\title{
Improved and customized secondary optics for photo-voltaic concentrators
}

\author{
Daniel Vázquez ${ }^{a}$, A. Álvarez Fernández-Balbuena ${ }^{a}$, Ángel García-Botella ${ }^{b}$, and Javier Alda ${ }^{a}$ \\ ${ }^{a}$ Applied Optics Complutense Group, University Complutense of Madrid, \\ Ave. Arcos de Jalón, 118. 28037 Madrid. Spain \\ ${ }^{b}$ Applied Optics Complutense Group, University Polytechnic of Madrid, \\ Ciudad Universitaria. 28040 Madrid. Spain
}

\begin{abstract}
In this contribution the line flow method is applied to an optimized secondary optics in a photovoltaic concentration system where the primary optics is already defined and characterized. This method is a particular application of photic field theory. This method uses the parameterization of a given primary optics, including actual tolerances of the manufacturing process. The design of the secondary optics is constrained by the selection of primary optics and maximizes the concentration at a previously specified collection area. The geometry of the secondary element is calculated by using a virtual source, which sends light in a first concentration step. This allows us to calculate the line flow for this specific case. This concept allows designing more compact and efficient secondary optics of photovoltaic systems.
\end{abstract}

Keywords: nonimaging optics, concentrators, photovoltaics, optical design

\section{INTRODUCTION}

Photovoltaic systems (PV) contain two main elements: a solar cell that converts optical power in electric power, and passive or active optical elements that redirect optical flux towards the solar cell. High-efficiency triplejunction solar cells require control of the irradiance distribution on the cell. The distribution has to be as uniform as possible, avoiding hot spots degrading the overall performance of the system. Solar concentrators are in charge of this delicate task. When no tracking system is available, a good behavior of the focusing optics is necessary for a wide range of incidence angle. To fulfill this specification, the design of the PV concentrators has developed several useful tools and ideas. In this sense, the concentration task can be shared by two optical elements. Primary optical system is used to gather as much flux as possible and redirect it towards the cell's localtion. The shape and design of this primary optics is of great importance to properly distribute the irradiance on the cell. On the other hand, this system can be complemented by another secondary element that helps the system to perform better. Secondary optics in a PV system is designed with different goals in mind. For example, improving the uniformity, enhancing the concentration, or selecting the operational wavelength are feasible objectives that can be fulfilled with this element. Primary optics redirect the irradiance towards the cell crossing a volume in front of the solar cell. Then, secondary optics should occupy part of this volume. ${ }^{1}$ There are many secondary optics types using several techniques with different complexity and cost. It is well known that a flow line concentrator can be used as a second stage concentrator in imaging and non-imaging optics. ${ }^{2,3}$ Dielectric CPCs have been developed by Winston, ${ }^{4}$ and its use has been analyzed deeply by Ning. ${ }^{5}$ A customized design of secondary optics needs the characterization of the irradiance distribution at the cell plane given by primary optics. Then, this distribution is modfied by designing the secondary optical element. This design should take into account the specification of manufacturing and mechanical and optical tolerances of primary optics. In figure 1 we show the actual light distribution obtained at the focal plane of a primary optical element having the shape of a plastic Fresnel lens made by injection moulding. Secondary optics should be placed between the Fresnel lens and the focal plane where the solar cell is placed.

Further author information: (Send correspondence to Daniel Vazquez)

Daniel Vazquez: E-mail: dvazquez@ucm.es, Telephone: +34.91.3946890

Nonimaging Optics: Efficient Design for Illumination and Solar Concentration XII,

edited by Roland Winston, Jeffrey M. Gordon, Proc. of SPIE Vol. 9572, 957205

(C) 2015 SPIE · CCC code: $0277-786 X / 15 / \$ 18 \cdot$ doi: $10.1117 / 12.2187962$

Proc. of SPIE Vol. 9572 957205-1 


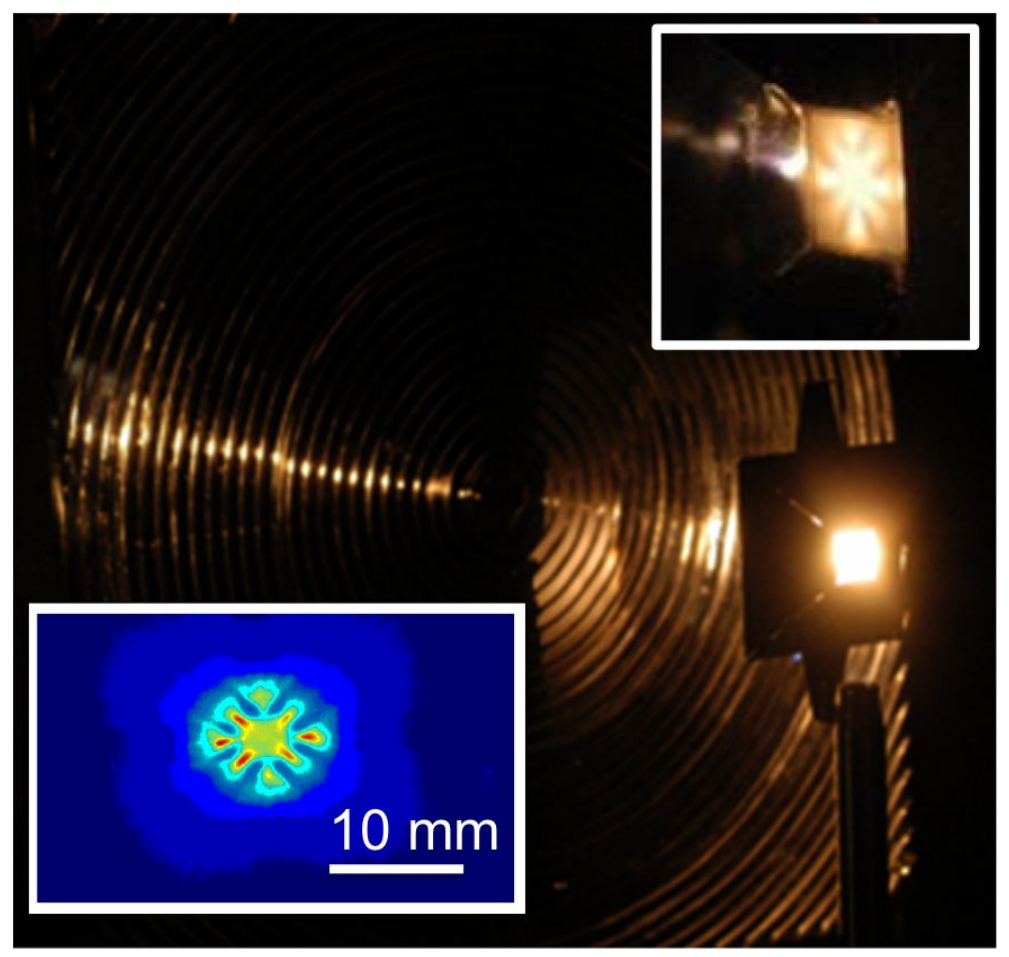

Figure 1. Experimental set-up for a primary optics Fresnel lens made of injected plastic. The inset at bottom left shows the actual irradiance distribution at the focal plane of the primary optics. The inset on top right shows a picture of this distribution.

Due to mechanical and optical tolerances, and aberrations, the actual performance of primary optics departs from the ideal design. Typically, the irradiance distribution is larger and less uniform than expected from simulations or analytical calculations. Here is where a customized secondary optics can improve the performance of primary optics and therefore increase the concentration ratio of the system.

There are many different devices proposed in the literature to improve and design secondary optics. A technique that has been used to optimized the geometry of a secondary optical element is the flow line method. ${ }^{6,7}$ Methods based on field theories are used in several areas of physics and engineering to optimize elements and systems. ${ }^{8}$ In radiometry and photometry, field-based methods use the concept of flux vector $\mathbf{J}$. The components of this vector, $\left(J_{x}, J_{y}, J_{z}\right)$ are the total light-flux per unit area which goes through the planes $y z, x z$ and $x y$, respectively at point $P$ in the space with coordinates $(x, y, z)$. In order to establish a complete light field theory, Gershum used the concept of photometric Light vectors. ${ }^{9,10}$

Gershun defined the light vector $D$ in the following way: at each point $P$ in space there is a vector, the light field. This is defined calculating the difference of illuminance between both sides of a plane element placed at $P$ and normal to the direction along with the field is defined. ${ }^{9}$ Later, Moon and Spencer ${ }^{10}$ defined the pharosage vector $D$ in this way: the magnitude of the pharosage vector is equal to the net radiant power per unit area at $P$, and the direction of the vector is the direction of the flow of the radiant energy at that point. Using the analogies between these definitions and the definition of geometrical vector flux, we have studied the design of concentrators within the frame of field theory.

Following Gershun and Moon for a space without radiation source, the phasorage vector obeys the following relation:

$$
\nabla D=0,
$$

being irrotational field, so

$$
\nabla \times D \neq 0
$$




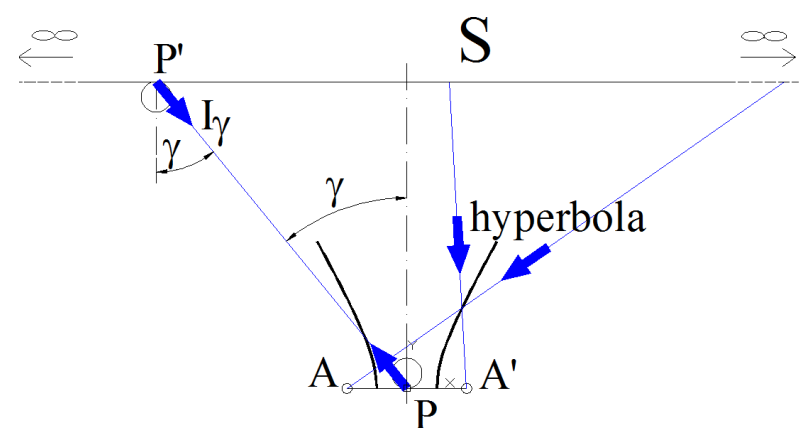

Figure 2. Geometrical definition of the hyperbola flux line between the plane of reception $P$ and the primary optics plane $S$. $A$ and $A^{\prime}$ are the focii of the hyperbolic flux lines.

There are only three irrotational field: those produced by a infinite uniform plane source, those produced by an infinite uniform cylinder and, those produced by a uniform sphere. In the case treated here we can find a number of geometries where the light field behaves as a quasipotential field, which satisfies

$$
D . \nabla \times D=0 .
$$

Rotationally symetric concentrators (symmetric in cylindrical coordinated systems), and translational concentrators (symmetric in rectangular coordinated systems) have been frequently used in practical designs. ${ }^{11}$ They have been also called 2D and 3D concentrators respectively. Those concentrators showing no symmetry in cylindrical coordinates systems nor Cartesian coordinates can be studied using field theory by using elliptical that preserve symmetry. ${ }^{8,12}$ As an example, the second stage hyperbolic system is a concentrator deeply studied even with a lens in its entry window and with several geometric variations. ${ }^{13}$

\section{CUSTOMIZATION OF CONCENTRATORS USING LINE FLOW}

Primary optics in a compound concentrator focus light on an area where the irradiance distribution can be measured and characterized. In this case, primary optics could be seen as a light source illuminating a reception plane. ${ }^{14,15}$ In an ideal case, the obtained photic field, or light field would be the same when a lambertian source placed at the reception plane sends light toward the primary optics, as it happesn with Inverse Line Flow Concentrators (ILFC).$^{16}$ In this contribution we have used a theoretical and perfect lambertian source. This source could describe how the primary optics actually sends light to the detector plane. In this last case the photic field would be better characterized and the calculation would be more accurate.

When a lambertian source strip $A A$ is considered, the geometry of the lines of optical flow are confocal hyperbolas with focii at $A$ and $A$ (see figure 2). Using light propagation reversibility we may study this problem from source to detector or from detector to source. ${ }^{16}$ In our case the same result is obtained when an infinite source $S$ is placed at a given distance to the strip, but only considering light arriving to $A A$ strip. If a point $P$ of strip $A A$ radiates an intensity $I$, in the direction $\gamma$ the infinite source will radiate the same intensity $I$, in the reversed direction (fsee figure 2).

If source $S$ is a finite source, which is the case when designing secondary optics for a PV system, the geometry of the flow lines changes and they will not be hyperbolic anymore as it happened in case of an infinite source (see figure 3.a). Miñano has addressed the problem from a different perspective in reference. ${ }^{17}$ In our case there is a part of the system that contains a dielectric tube or funnel. This tube can be quite close to the detector, or PV cell, even in direct contact. In those conditions the flow line method as it was shown in the previous case, is not directly applicable, as it happens in this paper.

When graphically calculating the flow lines (see figure 3.b), we find two different portions. In Zone A lines are stilly hyperbolic. However, in zone B the flow lines are not hyperbolic. Keeping this idea in mind we design the actual shape of an optimized or customized secondary optics. This optical surface is a diopter that separate the region behind the primary optics from the dielectric funnel ending at the PV cell. In an actual PV system, 


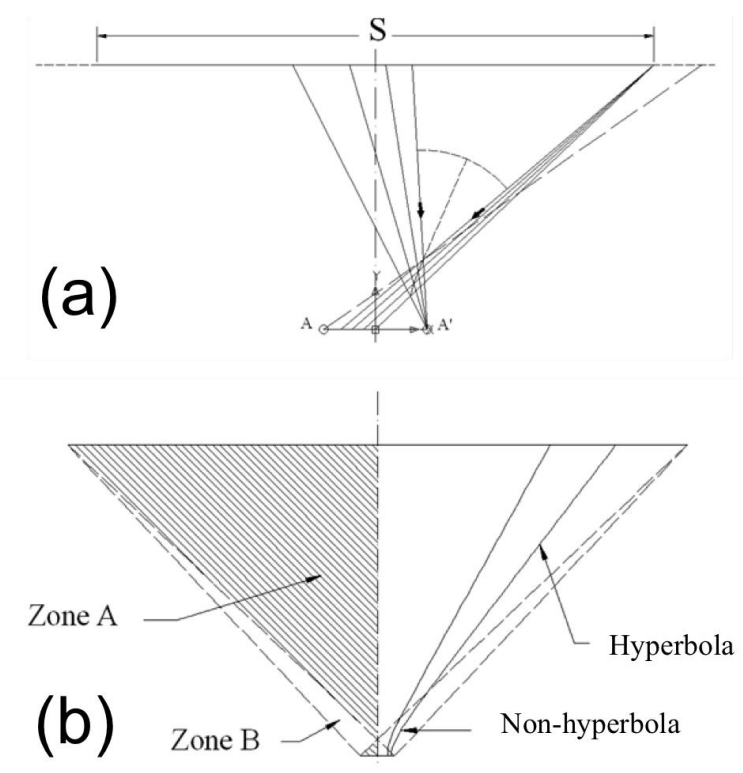

Figure 3. When the source is finite, there are regions of the funnel curve that are not hyperbolic.

primary optics produces a non-ideal uniform irradiance distribution as it required by the inverse finite source method. However, this is still a good approximation and we will maintain it. The actual shape of the diopter configuring the secondary optics is determined by analyzing the bending of light trajectories when crossing the interface between air and dielectric material. The equation governing this change is the Snell law

$$
n_{1} \sin \epsilon_{1}=n_{2} \sin \epsilon_{2},
$$

where $n_{1}$ and $n_{2}$ are the values of the index of refraction of air and dielectric, $\epsilon_{1}$ is the angle of incidence, and $\epsilon_{2}$ is the angle of refraction. Then, is is necessary to calculate the refracted photic field taking into account Snell law and the geoemtry of the refracting or front surface of the diopter. This is illustrated in figure 4. To do so, we first determine the location of point $P$ that is defined as the intersection of two lines departing from $A$ and $A^{\prime}$ and arriving to one of the limits of the primary optics plane and a given point $S$. This point $P$ moves when considering refraction by the outer furface of secondary optics. In this case it is necessary to calculate, for this point $P$ what is the ray that passing through point $P$ will be refracted in such way that it will go through the limits of the strip $A$ and $A$ (dashed lines). Using this geometical construction we can calculate the line flow at this point (see figure 4.c).

In figure 4.c we present the values of the geometric parameters of the secondary optics calculated using the line flux method. The system has a primary optics with a numeric aperture of 1 placed at a distance of $480 \mathrm{~mm}$ from the PV cell. The index of refraction of the dielectric is $n_{2}=1.52$, and the secondary optics diopter is an ellipse having semiaxis of $90 \mathrm{~mm}$ and $65,72 \mathrm{~mm}$. The profile of the flux line is shown also in figure 4.c

In this example we are using a Inverse Line flow Concentrator ILFC. Therefore, photic field is not for strip $A A$, and therefore the waist of the concentrator will not be in plane of this strip $A A$. In order to optimize the efficiency of the secondary optics we can avoid succesive reflections in the inner part of the dielectric funnel. To do that it will be necessary to make the system shorter than when considering more than one reflection.

\section{SIMULATION OF A REAL CASE}

In order to validate the concept and the proposed method we have evaluated a system using real raytracing. Light is emitted as collimated and misaligned at different angles with respect to the optical axis of the PV system. The calculation has been done within a circular area of $40 \mathrm{~mm}\left(A A^{\prime}=40 \mathrm{~mm}\right)$. The spot diagrams for each angle are calculated using 100000 rays, each one carrying $1 \mathrm{~W}$. The results, as an irradiance map is shown in figure 5 

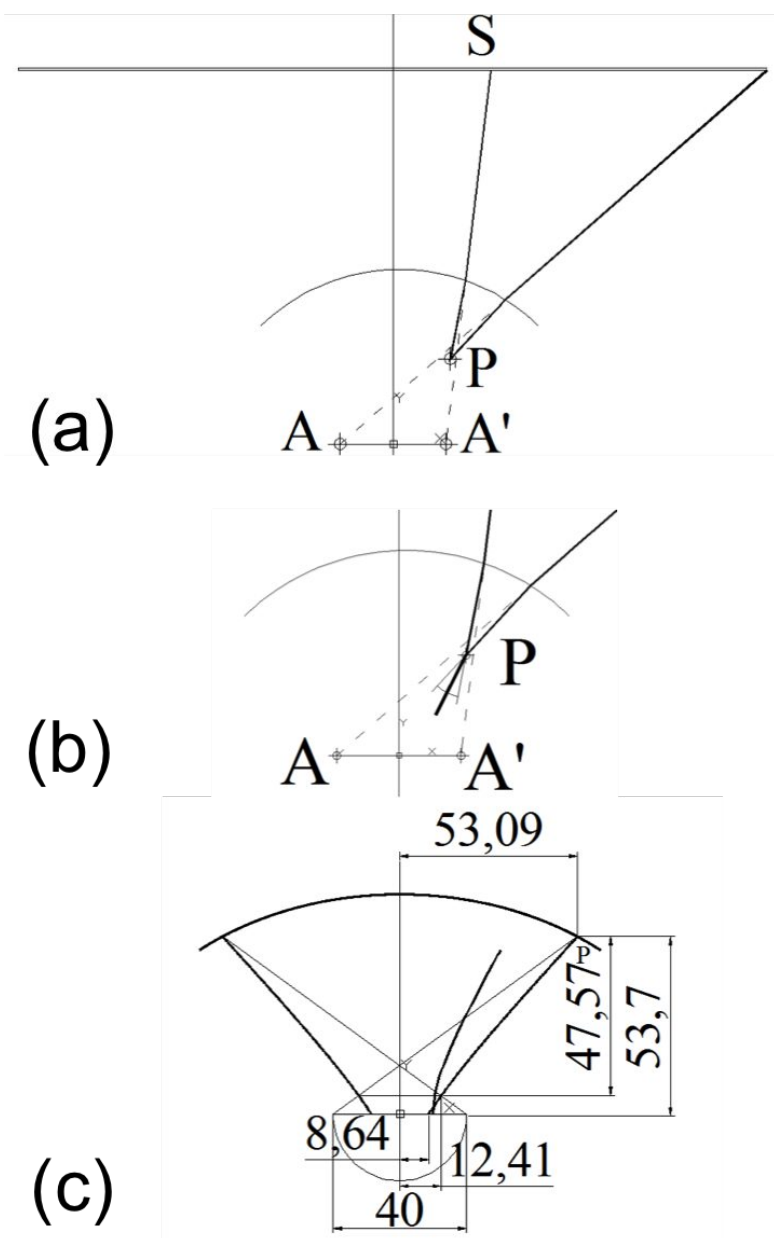

Figure 4. Secondary optics and line flux are defined by moving, using Snell law, the location of the point $P$ where the flux is defined.(a) Representation of point $P$ when considering point $S$ at the source. (b) Detail of the application of the Snell law that moves the location of point $P$ when introducing refractive secondary optics. (c) Geometrical parameters of a practical case of a PV cell having $4 \mathrm{~mm}$ in diameter (numbers are in $\mathrm{mm}$ ) 

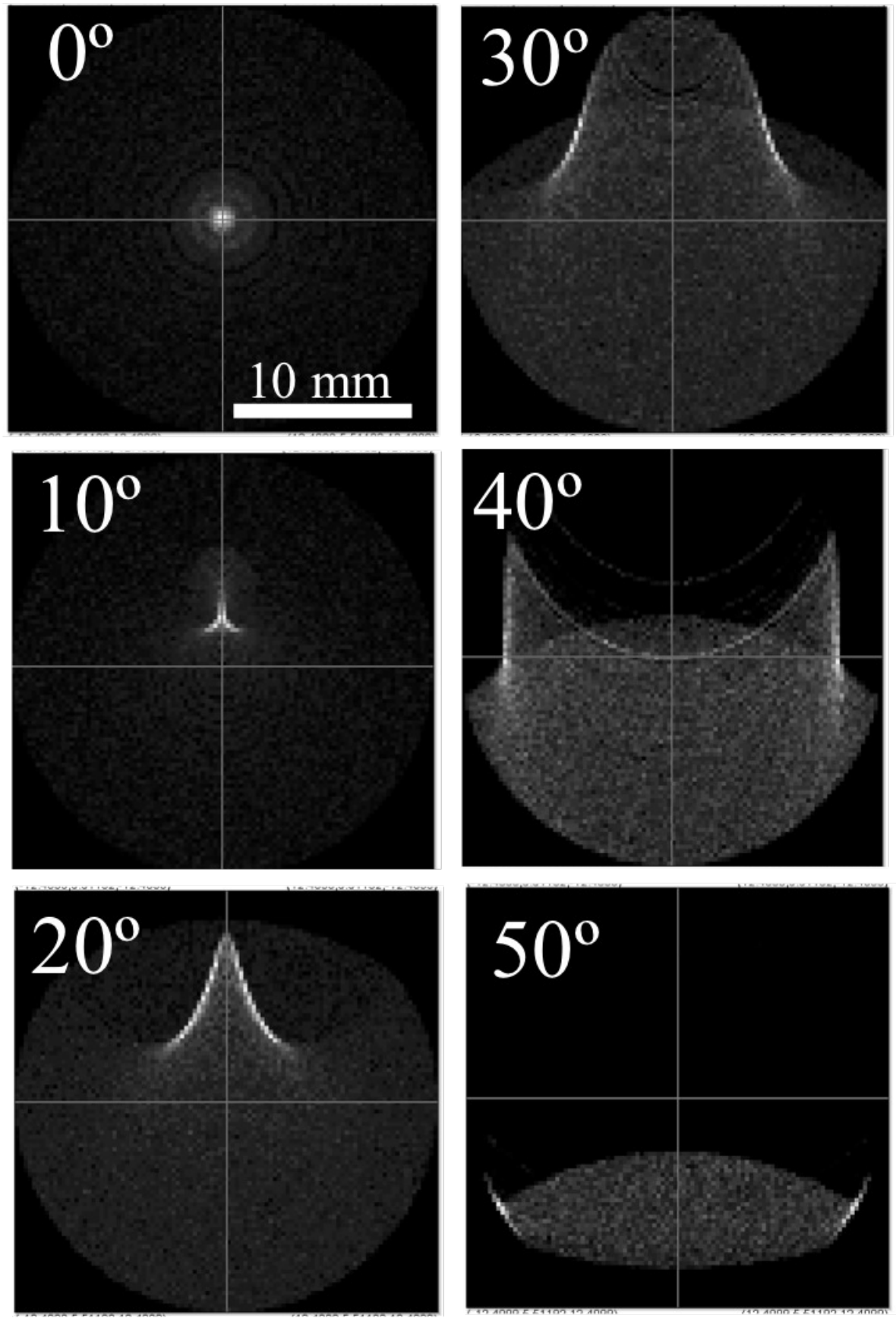

Figure 5. Irradiance maps at the location of the PV cell for an optimized secondary optics. Light is coming to the optical system from infinity at several angles of incidence, from $0^{\circ}$ to $50^{\circ}$. 


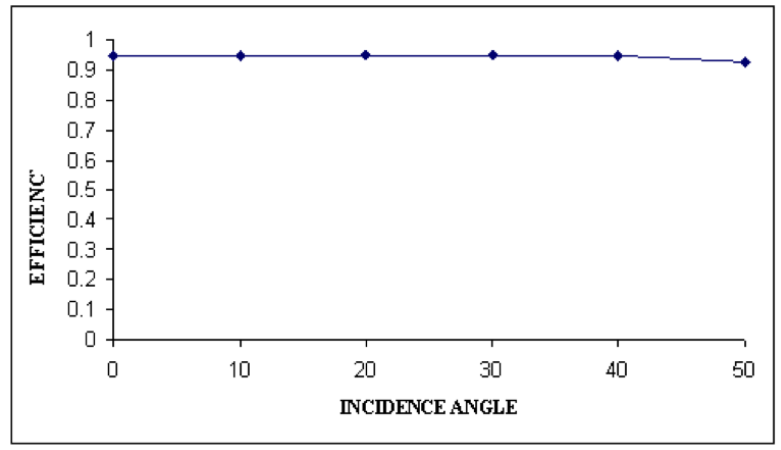

Figure 6. Efficiency of the system for different angles of incidence.
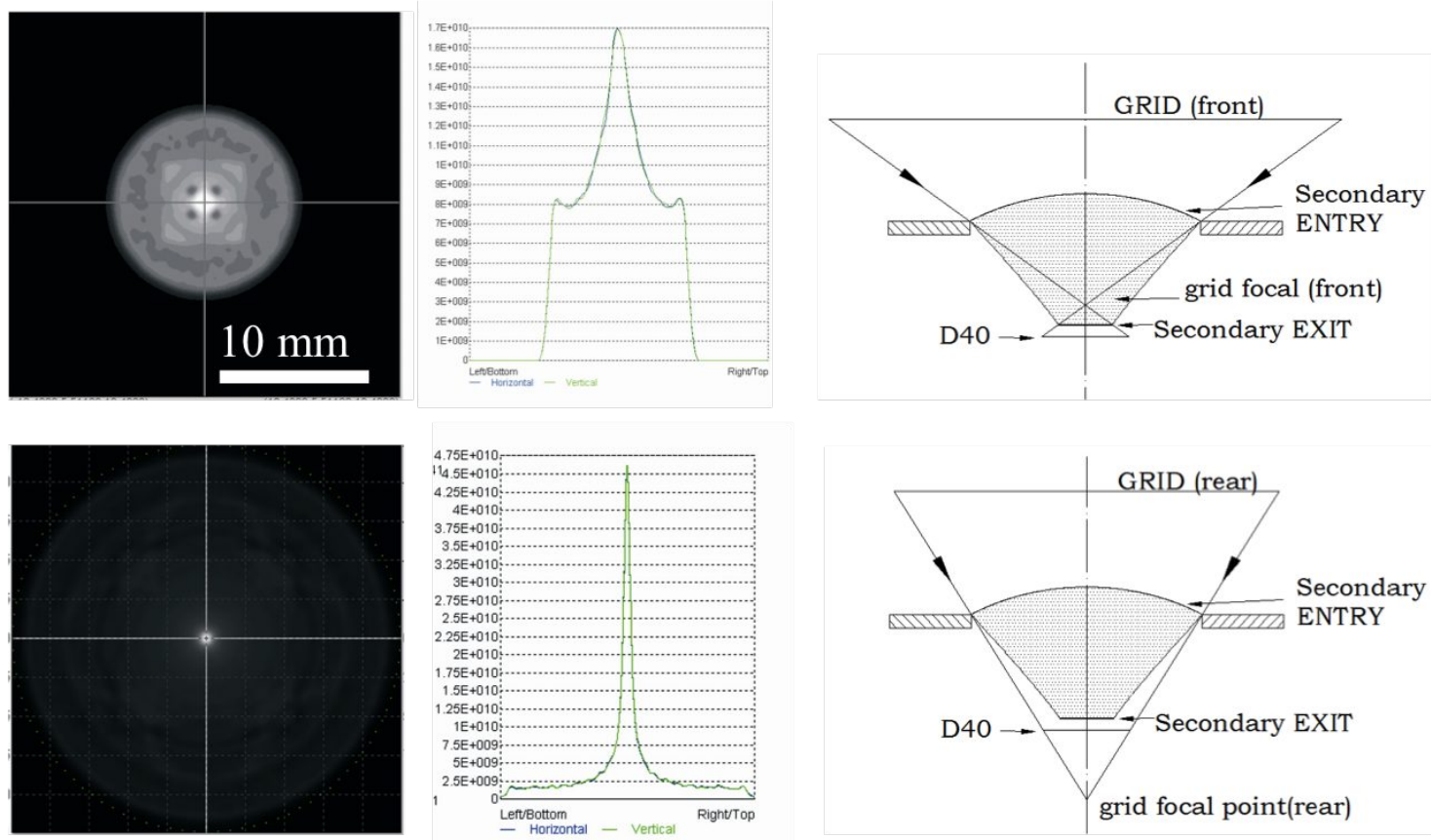

Figure 7. Irradiance maps (left), irradiance profile (center), and geometric configurations of two possible designs for a secondary optical element. The one on top focuses before the PV cell plane, and the one at the bottom focuses behind the PV cell plane.

A figure of merit is named as efficiency of the inverse line flow concentrator. This efficiency is defined as the ratio between the energy on the detector plane, to the energy in the plane where strip $A A$ is. We should remember that the purpose of secondary optics is to concentrate light sent to $A A^{\prime}$ by the primary optics. We have done this calculation for incidence angles from $0^{\circ}$ to $50^{\circ}$. This last value 50 is above the maximum angle limited by the design using an aperture $\mathrm{F} \#=1$. Figure 6 shows the value of this efficiency that remains very close to 1 for the whole angular range. Then, we may conclude that secondary optics is behaving properly.

Finally, we have simulated a system which has a primary optics, having a Fresnel design, that is collimating light onto the secondary input surface. We have analyzed two different configurations One of them focus before strip $A A$, and the other focus behind this strip. In the first case the efficiency is $94,76 \%$ and in the second case is $93.16 \%$. The irradiance map and simulations are shown in figure 7 . 


\section{CONCLUSIONS}

In this work a method to design secondary optics for PV system based on line flow method, the Inverse Line Flow Method, has been developed. This design methodology can be applied for any given and fabricated primary optics with their own fabrication tolerances. Therefore, this design method can be applied when the energy onto concentration plane has been measured or simulated. This characterization can be made after fabricating primary optics allowing customization. The secondary optics obtained with ILFC is more compact than those obtained by line flow method.

\section{ACKNOWLEDGMENTS}

This work has been supported by the Ministerio de Economia y Competitividad of Spain through the project TEC2013-40442

\section{REFERENCES}

[1] Vazquez, D., Alvarez, A., Bernabeu, E., Munoz, J., Domingo, A., and Garcia, A., "New concentrador multifocal fresnel lens for improved uniformity design and characterization," in [Proc SPIE Novel optical design and optimization XII], 7407, 740701 (2009).

[2] Winston, R. and Welford, W. T., "Design of nonimaging concentrators as second stages in tandem with image-forming first-stage concentrators," Appl. Opt. 19, 347-351 (1980).

[3] O'Gallagher, J. and Winston, R., "Axially symmetric nonimaging flux concentrators with the maximum theoretical concentration ratio," J. Opt. Soc. Am. A 4, 123-124 (1987).

[4] Winston, R., "Dielectric compound parabolic concentrators," Appl. Opt. 15(2), 291-292 (1976).

[5] Ning, X., Winston, R., and O'Gallaher, J., "Dielectric totally internally reflecting concentrators," Appl. Opt. 26(2), 300-305 (1987).

[6] Winston, R. and Welford, W. T., "Geometrical vector flux and some new nonimaging concentrators," $J$. Opt. Soc. Am. A 69(4), 532-536 (1979).

[7] Winston, R. and Welford, W., "Ideal flux concentrators as shapes that do not disturb the geometrical vector flux field: A new derivation of the compound parabolic concentrator," J. Opt. Soc. Am. A 69(4), 536-539 (1979).

[8] Moon, P. and Spencer, D. E., [Field theory handbook], Springer-Verlag (1988).

[9] Gershun, A., "The light field," J. Math. Phys, XVIII, 51-151 (1939).

[10] Moon, P. and Spencer, D. E., [Photic Field], Massachusetts Institute of Technology Press (1981).

[11] Winston, R., Minano, J. C., and Benitez, P., [Nonimaging optics], Elsevier Academic Press, New York (2005).

[12] Garcia, A., Alvarez, A., Vazquez, D., and Bernabeu, E., "Hiperbolic parabolic concentrator (hpc)," Appl. Opt. 48(4), 712-715 (2009).

[13] Garcia, A., Alvarez, A., Vazquez, D., and Bernabeu, E., "Field method for concentrador design," in [Proc. SPIE Novel optical design and optimization XII], 7423, 742307 (2009).

[14] Ries, H. R. and Winston, R., "Tailored edge ray reflector for illumination," J. Opt. Soc. Am. A 32(13), 2243-2251 (1994).

[15] Gordon, J. M. and Ries, H. R., "Tailored edge ray concentratorsas ideal second stages for fresnel lens reflectors," Appl. Opt. 32(13), 2243-2251 (1993).

[16] Leutz, R. and Annen, H. P., "Reverse ray-tracing model for the performance evaluation of stationary solar concentrators," Solar Energy 2007, 761-767 (2007).

[17] Minano, J. C., "Design and optimal and ideal 2-d concentrator with the collector immersed in a dielectric tube," Appl. Opt. 22(24), 3960-3965 (1985). 